\title{
Iterative Learning Applied to Hydraulic Pressure Control
}

\author{
P. H. Gøytil ${ }^{1}$ M.R. Hansen ${ }^{1}$ G. Hovland ${ }^{1}$ \\ ${ }^{1}$ Mechatronics Group, University of Agder, N-4898 Grimstad, Norway.
}

\begin{abstract}
This paper addresses a performance limiting phenomenon that may occur in the pressure control of hydraulic actuators subjected to external velocity disturbances. It is demonstrated that under certain conditions a severe peaking of the control error may be observed that significantly degrades the performance of the system due to the presence of nonlinearities. The phenomenon is investigated numerically and experimentally using a system that requires pressure control of two hydraulic cylinders. It is demonstrated that the common solution of feed forwarding the velocity disturbance is not effective in reducing the peaking that occurs as a result of this phenomenon. To improve the system performance, a combination of feedback and iterative learning control (ILC) is proposed and evaluated. The operating conditions require that ILC be applied in combination with a feedback controller, however the experimental system inherently suffers from limit cycle oscillations under feedback due to the presence of valve hysteresis. For this reason the ILC is applied in combination with a feedback controller designed to eliminate limit cycle oscillations based on describing function analysis. Experimental results demonstrate the efficacy of the solution where the feedback controller successfully eliminates limit cycle oscillations and the ILC greatly reduces the peaking of the control error with reductions in the RMS and peak-to-peak amplitude of the error by factors of more than 30 and 19, respectively. Stability of the proposed solution is demonstrated analytically in the frequency domain and verified on the experimental system for long periods of continuous operation.
\end{abstract}

Keywords: Hydraulic pressure control, peaking phenomenon, iterative learning control, limit cycles.

\section{Introduction}

Hydraulic actuators are used in a wide range of applications, for example mobile machinery, offshore drilling and material handling. In general, hydraulic actuators are used whenever high power density is of major importance. Both open-loop and closed-loop control systems are common, depending on the system requirements. Closed-loop control may be further divided into two categories: motion control and pressure control. Motion control refers to position and velocity control, whereas pressure control, in the context of this paper, refers to controlling the pressure difference across the actuator, which includes force control of linear actuators and torque control of rotary actuators.
Hydraulic pressure control is a challenging and one of the most studied problems within the field of hydraulic control (Ledezma et al., 2015), often requiring system modifications or sophisticated control methods even for simple control specifications (Baghestan et al., 2014). Several challenges unique to hydraulic pressure control arise from the effects of an inherent feedback path in the system's dynamics coupling the pressures in the chambers of the actuator to its velocity, commonly known as the natural velocity feedback (Zhao et al., 2004). When the controlled actuator force or torque is applied to an environment whose natural frequency is not significantly above that of the valve actuator combination, the system's frequency response suffers from an antiresonance at the natural frequency 
of the environment followed by a resonant mode. This phenomenon makes high bandwidth tracking of pressure controlled actuators a difficult task that has been studied extensively, see for example (Zhao et al., 2004) and (Lamming et al., 2010).

Another consequence of the natural velocity feedback is the effect of external velocity disturbances in pressure controlled hydraulic actuators. Due to the near incompressibility of hydraulic oil and the coupling between the pressures of the actuator and its velocity, even minor velocity disturbances may correspond to significant disturbances in the pressures of the actuator and thus in the controlled output (Esfandiari and Sepehri, 2014). A common method of improving the performance in situations where velocity disturbances are measurable involves feed forwarding from the disturbance, see for example (Conrad and Jensen, 1987) and (Jiao et al., 2004). As will be seen in Sections 4 and 5 however, a large abrupt peaking of the control error may occur under certain operation conditions that is not reduced by feed forwarding from the velocity disturbance and appears to be a previously unaddressed phenomenon.

This paper examines the conditions under which this phenomenon occurs and several factors that may reduce or amplify the resulting peaking of the control error are identified. The relevance of the phenomenon from a system design perspective is discussed and the use of iterative learning control (ILC) is proposed and evaluated as a potential solution for achieving satisfactory system performance. An additional contribution of the paper is the application of ILC in a closed-loop fashion to a system that inherently suffers from limit cycle oscillations under feedback.

For systems performing repetitive tasks, ILC has been shown to be capable of significantly improving the system's performance (Blanken et al., 2017), with several industrial applications having been reported (Boeren et al., 2016). Originating from the field of industrial robotics where industrial manipulators are often used to perform the same tasks repetitively (Wallén, 2011), ILC takes advantage of the repetitive nature of the system in order to improve the performance iteratively. By recording the system performance each iteration, a learning algorithm updates a feed forward signal that is either sent to the plant input or modifies the set point or reference trajectory of a conventional feedback controller (Longman, 2000). Figure 1 illustrates a simple example of an ILC algorithm where the output of the ILC is denoted $u$. The block $G$ represents the dynamics of a plant and its feedback controller. The reference input is $r(t)$ and the output of the plant is denoted $y(t)$, where the subscript $k$ denotes iteration. By recording the control error $e(t)$ in each iteration and feed forwarding it to the next iteration, the control error is reduced provided that the behaviour of the system is deterministic. The ILC algorithm in this example may be thought of as a feed forward in the time domain, and a feedback P-controller with unity gain in the iteration domain. This outlines the basic mechanism of an ILC controller, where more sophisticated algorithms are found in the literature, see for example (Wallén, 2011).

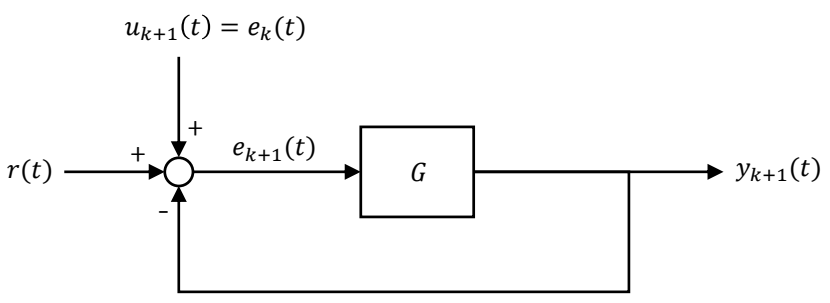

Figure 1: Basic mechanism of an ILC configuration.

The rest of the paper is organized as follows. Section 2 describes the experimental system and its control requirements. Modelling of the system is presented in Section 3 and an investigation into the aforementioned peaking phenomenon is conducted in Section 4. Sections 5 and 6 concern the control designs to be evaluated with experimental results given in Section 7. Lastly, a discussion and conclusions are found in Section 8 .

\section{System Description}

The experimental system is a test rig designed for studying friction phenomena and friction compensation methods in hydraulic cylinders, see Figure 2. The primary component of the test rig is the main cylinder with a piston diameter of $125 \mathrm{~mm}$, which is controlled by means of a servo valve and may translate freely in the vertical direction. In addition, two hydraulic cylinders connected in parallel, referred to as the rotation cylinders, may be used to rotate the piston rod and piston of the main cylinder without affecting the extension or retraction of the main cylinder. As the main cylinder is actuated in the vertical direction, the rotation cylinders simply follow the main cylinder as they glide freely on low-friction sliding tracks. This arrangement constitutes a non-model based friction compensation method that was studied in (Ottestad et al., 2012).

Further, two additional cylinders, referred to as the load cylinders, connected in parallel hydraulically are oriented opposite and attached to the main cylinder mechanically. A second servo valve, connected to the 
load cylinder circuit may be used to control the pressure differential across the load cylinders and thus the force applied to the main cylinder. The purpose of the load cylinders is to simulate the application of external forces applied to the main cylinder. Due to their mechanical connection, any actuation of the main cylinder forces the load cylinders to move as well and therefore represents an external velocity disturbance to the load cylinder circuit.

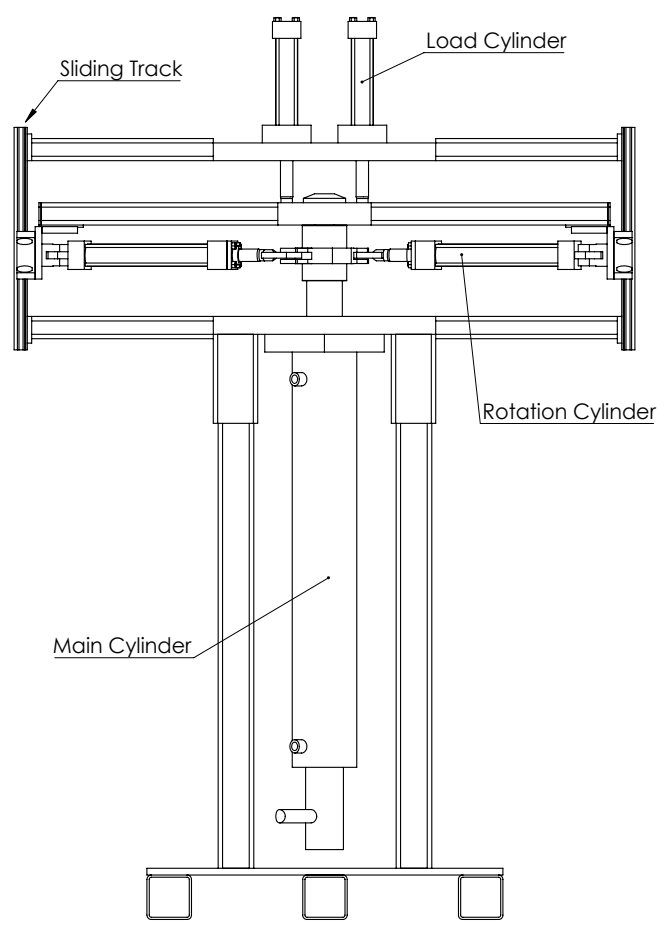

Figure 2: Overview of the experimental system.

The work presented in this paper concerns the design of a force controller for the load cylinder circuit, where for the application under consideration the load cylinders are required to maintain a constant force of $10 \mathrm{kN}$ in the presence of external velocity disturbances generated by the main cylinder. In particular, the load cylinders should be capable of maintaining their force set point with a reasonable accuracy as the main cylinder is actuated in a sinusoidal velocity profile with a frequency of $0.1 \mathrm{~Hz}$ and amplitudes of up to $40 \mathrm{~mm}$, where the worst case situation, i.e. an amplitude of 40 $\mathrm{mm}$ is considered here. The remaining control hardware consists of a CompactRIO from National Instruments that is connected to the servo valves and the instrumentation of the system, used for data acquisition and the implementation of control algorithms in the LabVIEW programming environment.

The rest of the paper concerns the control design of the load cylinder circuit for which the use of the rota- tion cylinders is not relevant and therefore not considered here.

\section{Modelling}

In this section a linear transfer function is derived for the load cylinder circuit for use in the control design. First, the governing equations of the system are established. Next, the nonlinear equations are linearized and combined to describe the dynamics from servo valve input to the output force $F_{L C}$.

Due to the large size of the fluid volumes in the main cylinder chambers, the flexibility of the main cylinder has to be accounted for. The load cylinders are therefore modelled as applying their output force to a massspring-damper system as shown in Figure 3. The mass $m$ refers to the lumped mass of all parts that may be accelerated by the load cylinder circuit, the spring stiffness $k$ describes the stiffness of the main cylinder and the damping coefficient $d$ is due to the presence of viscous damping.

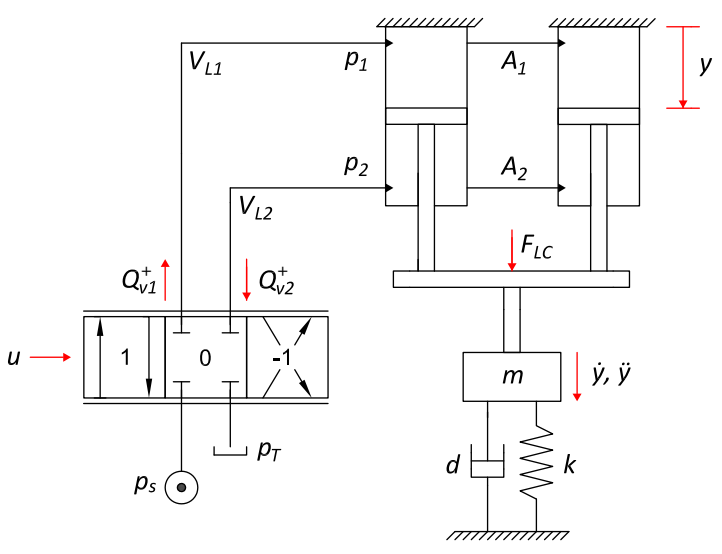

Figure 3: Modelling of the load cylinder circuit.

The load cylinder circuit is equipped with hydraulic pressure sensors in both the piston-side and rod-side chambers, however no force transducer is available. For this reason the friction of the load cylinder circuit, which is negligible in comparison to the required output force, is not included in the estimation of the force applied by the load cylinder circuit.

The fluid volumes whose pressures are denoted $p_{1}$ and $p_{2}$ are referred to as the first and second control volumes, respectively. The volume flows of the servo valve are described by the orifice equation (Merritt, 
1967). For extension $(u \geq 0)$ :

$$
\begin{aligned}
& Q_{v 1}=C_{d} \cdot A_{d 0} \cdot u \cdot \sqrt{\frac{2}{\rho} \cdot\left(p_{s}-p_{1}\right)} \\
& Q_{v 2}=C_{d} \cdot A_{d 0} \cdot u \cdot \sqrt{\frac{2}{\rho} \cdot\left(p_{2}-p_{T}\right)}
\end{aligned}
$$

For retraction $(u<0)$ :

$$
\begin{aligned}
& Q_{v 1}=C_{d} \cdot A_{d 0} \cdot u \cdot \sqrt{\frac{2}{\rho} \cdot\left(p_{1}-p_{T}\right)} \\
& Q_{v 2}=C_{d} \cdot A_{d 0} \cdot u \cdot \sqrt{\frac{2}{\rho} \cdot\left(p_{s}-p_{2}\right)}
\end{aligned}
$$

The volume in each control volume is described by:

$$
\begin{aligned}
& V_{1}=V_{L 1}+A_{1} \cdot y \\
& V_{2}=V_{L 2}+A_{2} \cdot(h-y)
\end{aligned}
$$

Applying the continuity equation to each control volume:

$$
\begin{aligned}
& Q_{v 1}=A_{1} \cdot \dot{y}+\frac{V_{1}}{\beta} \cdot \dot{p_{1}} \\
& Q_{v 2}=A_{2} \cdot \dot{y}-\frac{V_{2}}{\beta} \cdot \dot{p_{2}}
\end{aligned}
$$

From Pascal's law, neglecting friction:

$$
F_{L C}=p_{1} \cdot A_{1}-p_{2} \cdot A_{2}
$$

Applying Newton's second law to the mass $m$ :

$$
m \cdot \ddot{y}=p_{1} \cdot A_{1}-p_{2} \cdot A_{2}-d \cdot \dot{y}-k \cdot y
$$

Linearizing the orifice equation using a Taylor series expansion and ignoring higher order terms:

$$
\begin{aligned}
& Q_{v 1}=K_{q u 1} \cdot u-K_{q p 1} \cdot p_{1} \\
& Q_{v 2}=K_{q u 2} \cdot u+K_{q p 2} \cdot p_{2}
\end{aligned}
$$

The linearization coefficients for extension are given by $(u \geq 0)$ :

$$
\begin{aligned}
& K_{q u 1, e x t}=C_{d} \cdot A_{d 0} \cdot \sqrt{\frac{2}{\rho} \cdot\left(p_{s}-p_{1 s s}\right)} \\
& K_{q u 2, e x t}=C_{d} \cdot A_{d 0} \cdot \sqrt{\frac{2}{\rho} \cdot\left(p_{2 s s}-p_{T}\right)} \\
& K_{q p 1, e x t}=\frac{C_{d} \cdot A_{d 0} \cdot u_{s s}}{\sqrt{2 \cdot \rho \cdot\left(p_{s}-p_{1 s s}\right)}} \\
& K_{q p 2, e x t}=\frac{C_{d} \cdot A_{d 0} \cdot u_{s s}}{\sqrt{2 \cdot \rho \cdot\left(p_{2 s s}-p_{T}\right)}}
\end{aligned}
$$

The linearization coefficients for retraction are given by $(u<0)$ :

$$
\begin{aligned}
& K_{q u 1, r e t}=C_{d} \cdot A_{d 0} \cdot \sqrt{\frac{2}{\rho} \cdot\left(p_{1 s s}-p_{T}\right)} \\
& K_{q u 2, r e t}=C_{d} \cdot A_{d 0} \cdot \sqrt{\frac{2}{\rho} \cdot\left(p_{s}-p_{2 s s}\right)} \\
& K_{q p 1, r e t}=-\frac{C_{d} \cdot A_{d 0} \cdot u_{s s}}{\sqrt{2 \cdot \rho \cdot\left(p_{1 s s}-p_{T}\right)}} \\
& K_{q p 2, r e t}=-\frac{C_{d} \cdot A_{d 0} \cdot u_{s s}}{\sqrt{2 \cdot \rho \cdot\left(p_{s}-p_{2 s s}\right)}}
\end{aligned}
$$

Taking Laplace transforms and combining Eqs. (7)(12), the following transfer function is derived describing the dynamics from servo valve input to the force applied by the load cylinders using block diagram methods:

$$
G_{L C}(s)=\frac{F_{L C}(s)}{u(s)}=G_{v}(s) \cdot G_{h y d}(s)
$$

where:

$G_{h y d}(s)=\frac{n_{3} \cdot s^{3}+n_{2} \cdot s^{2}+n_{1} \cdot s+n_{0}}{d_{4} \cdot s^{4}+d_{3} \cdot s^{3}+d_{2} \cdot s^{2}+d_{1} \cdot s+d_{0}}$

and:

$$
\begin{aligned}
n_{3}= & A_{1} K_{q u 1} V_{2} \beta m+A_{2} K_{q u 2} V_{1} \beta m \\
n_{2}= & A_{1} K_{q u 1} V_{2} \beta d+A_{2} K_{q u 2} V_{1} \beta d \\
& +A_{1} K_{q p 2} K_{q u 1} \beta^{2} m+A_{2} K_{q p 1} K_{q u 2} \beta^{2} m \\
n_{1}= & A_{1} K_{q u 1} V_{2} \beta k+A_{2} K_{q u 2} V_{1} \beta k \\
& +A_{1} K_{q p 2} K_{q u 1} \beta^{2} d+A_{2} K_{q p 1} K_{q u 2} \beta^{2} d \\
n_{0}= & A_{1} K_{q p 2} K_{q u 1} \beta^{2} k+A_{2} K_{q p 1} K_{q u 2} \beta^{2} k
\end{aligned}
$$

and:

$$
\begin{aligned}
d_{4}= & V_{1} V_{2} m \\
d_{3}= & V_{1} V_{2} d+K_{q p 1} V_{2} \beta m+K_{q p 2} V_{1} \beta m \\
d_{2}= & V_{1} V_{2} k+A_{1}^{2} V_{2} \beta+A_{2}^{2} V_{1} \beta+K_{q p 1} K_{q p 2} \beta^{2} m \\
& +K_{q p 1} V_{2} \beta d+K_{q p 2} V_{1} \beta d \\
d_{1}= & A_{1}^{2} K_{q p 2} \beta^{2}+A_{2}^{2} K_{q p 1} \beta^{2}+K_{q p 1} K_{q p 2} \beta^{2} d \\
& +K_{q p 1} V_{2} \beta k+K_{q p 2} V_{1} \beta k \\
d_{0}= & K_{q p 1} K_{q p 2} \beta^{2} k
\end{aligned}
$$

The servo valve dynamics are modelled as a second order transfer function from valve input command to output spool position $u$ :

$$
G_{v}(s)=\frac{u(s)}{u_{r e f}(s)}=\frac{1}{\frac{1}{\omega_{v}^{2}} s^{2}+\frac{2 \zeta_{v}}{\omega_{v}} s+1}
$$

The parameters of the operating point considered as the worst case in terms of stability have been identified 
Table 1: Overview of system parameters.

\begin{tabular}{cll}
\hline Parameter & Value & Unit \\
\hline$K_{q u 1}$ & $1.0467 \cdot 10^{-3}$ & $\mathrm{~m}^{3} / \mathrm{s}$ \\
$K_{q u 2}$ & $1.1217 \cdot 10^{-3}$ & $\mathrm{~m}^{3} / \mathrm{s}$ \\
$K_{q p 1}$ & $2.05 \cdot 10^{-12}$ & $\left.\mathrm{~m}^{3} / \mathrm{s} \cdot \mathrm{Pa}\right)$ \\
$K_{q p 2}$ & $1.53 \cdot 10^{-12}$ & $\mathrm{~m}^{3} /(\mathrm{s} \cdot \mathrm{Pa})$ \\
$\beta$ & $10^{9}$ & $\mathrm{~Pa}$ \\
$k$ & $2.4 \cdot 10^{7}$ & $\mathrm{~N} / \mathrm{m}$ \\
$m$ & 98.4 & $\mathrm{~kg}$ \\
$d$ & 500 & $\mathrm{~N} \cdot \mathrm{s} / \mathrm{m}$ \\
$A_{1}$ & 0.025 & $\mathrm{~m}^{2}$ \\
$A_{2}$ & 0.013 & $\mathrm{~m}^{2}$ \\
$V_{1}$ & $2.359 \cdot 10^{-4}$ & $\mathrm{~m}^{3}$ \\
$V_{2}$ & $2.361 \cdot 10^{-4}$ & $\mathrm{~m}^{3}$ \\
$\zeta_{v}$ & 0.85 & - \\
$\omega_{v}$ & 91.4 & $\mathrm{~Hz}$ \\
\hline
\end{tabular}

in (Gøytil, 2017) as given by Table 1 and are used in the control design together with the linearized model.

Additionally, for the numerical investigations presented in the following section, a simulation model of the experimental system is constructed using library elements found in the commercially available software package SimulationX as shown in Figure 4. In this model the velocity control of the main cylinder is considered ideal and for this reason the motion of the main cylinder is implemented as a preset that simply forces the load cylinders to move with the sinusoidal velocity profile of the main cylinder. As in the mathematical model, the elasticity of the main cylinder fluid column is represented by a mass-spring-damper system. In addition, five orifices, not shown in Figure 4 are used to model the leakage flows of the servo valve which are assumed to be laminar and modelled based on the null leakage of the servo valve that was measured in (Gøytil, 2017).

\section{Nonlinear Peaking Phenomenon}

This section investigates a performance limiting phenomenon that may be observed in pressure controlled hydraulic actuators subjected to external velocity disturbances. As will be seen in Section 5, the load cylinder circuit suffers from a severe peaking of the control error at the instances where the velocity disturbance from the main cylinder forces the load cylinders to change direction. In this paper this is referred to as the nonlinear peaking phenomenon and is reported and investigated here using an asymmetrical actuator. This appears to be a previously unaddressed phenomenon,

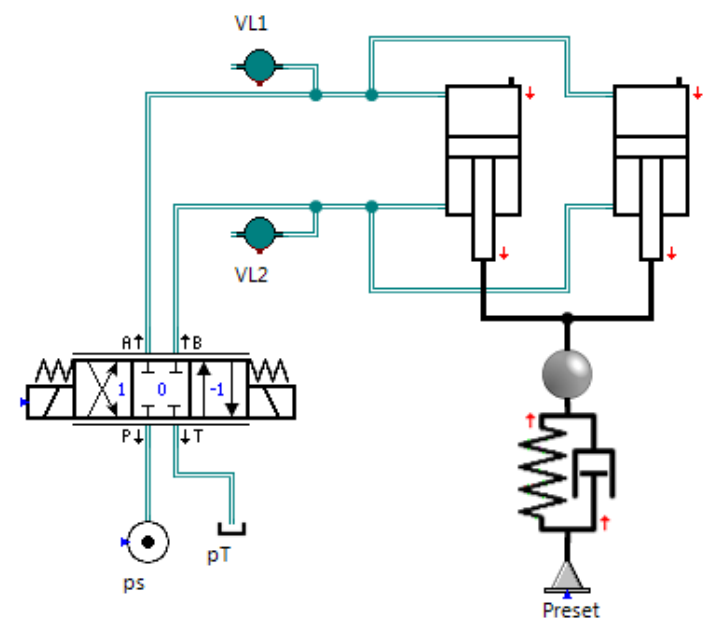

Figure 4: Numerical simulation model constructed using SimulationX.

although it may be observed in the experimental work of (Klausen and Tørdal, 2015) for a symmetrical actuator. In (Jiao et al., 2004) on the other hand, the absence of this phenomenon may be observed both numerically and experimentally also using a symmetrical actuator, despite the presence of external velocity disturbances that periodically force the actuator to change direction, indicating that the appearance of the nonlinear peaking phenomenon is dependent upon the system configuration. This section presents numerical results demonstrating some of the factors that determine whether or not this phenomenon occurs for a given system configuration as well as several factors that may amplify the magnitude of this peaking.

Using the numerical simulation model developed in the previous section, Figure 5 shows the control error of the load cylinder circuit using the PI controller of Section 5 in the presence of a sinusoidal velocity disturbance with an amplitude of $10 \mathrm{~mm}$ and a frequency of $0.1 \mathrm{~Hz}$ for a force set point of $0 \mathrm{kN}$. The solid line in Figure 5 indicates the control error for feedback control alone and the dashed line indicates the control error for feedback combined with a fixed-gain feed forward (ff) from the velocity disturbance. Observe in Figure 5 that for this sinusoidal velocity disturbance, the control error appears sinusoidal, as may also seen in the numerical and experimental work of (Jiao et al., 2004). Furthermore, a significant reduction in the control error is achieved by feed forwarding the velocity disturbance, where the peak-to-peak amplitude of the control error is reduced from $521 \mathrm{~N}$ to $25 \mathrm{~N}$ after adding the feed forward term to the controller.

Next, the force set point is changed from $0 \mathrm{kN}$ to 10 $\mathrm{kN}$, while all other parameters of the simulation model 
and the velocity disturbance are kept the same. Figure 6 shows the control error for this situation. Observe the change in the overall shape of the control error both with and without the use of feed forward. In Figure 6 an abrupt peaking of low magnitude is observed when the external velocity disturbance forces the load cylinders to change direction. Under these conditions, the control error reaches a peak-to-peak amplitude of 797 $\mathrm{N}$ using feedback and $226 \mathrm{~N}$ using feedback combined with feed forward, where the feed forward gain was adjusted slightly for optimal performance after changing the force set point.

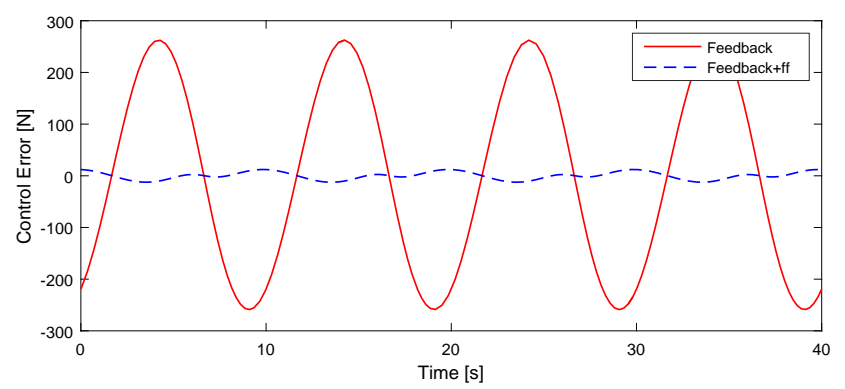

Figure 5: Force set point of $0 \mathrm{kN}$.

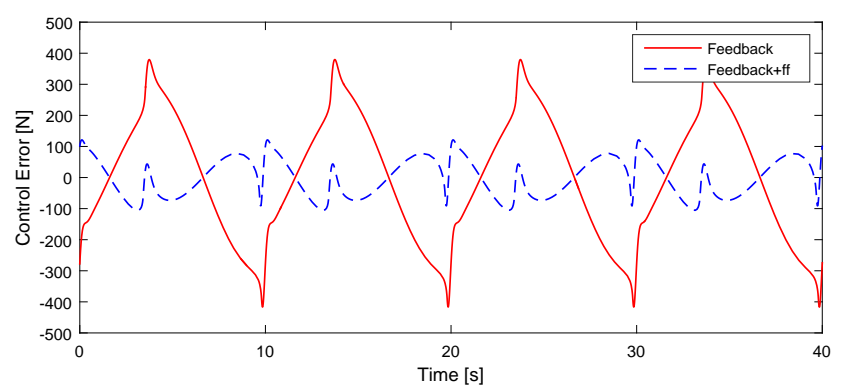

Figure 6: Force set point of $10 \mathrm{kN}$.

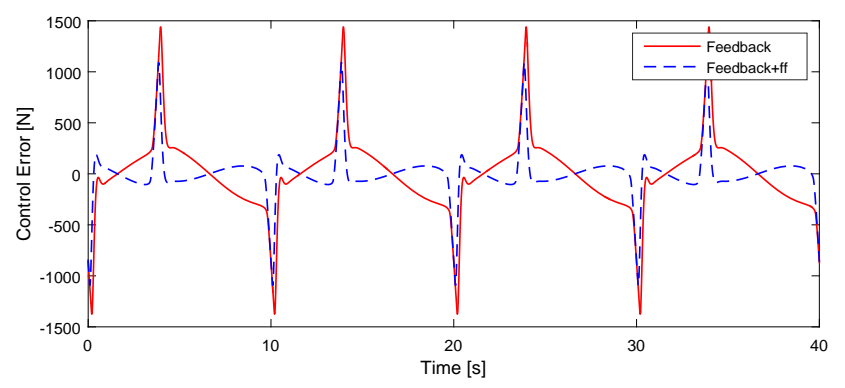

Figure 7: Force set point of $10 \mathrm{kN}$ and a valve deadband of $0.5 \%$.
Observe that although the feed forward is able to improve the overall accuracy, the peaking that occurs as the load cylinders change directions appear to be of the same magnitude. This is the phenomenon referred to in this paper as the nonlinear peaking phenomenon. The reason for the appearance of the nonlinear peaking phenomenon in Figure 6 after increasing the force set point becomes clear upon inspecting how the change in the force set point affects the states of the system: in Figure 5, the velocity disturbance and force set point for the current system configuration results in the servo valve operating only about one side of valve null, in this case $u<0$. This is determined by a number of factors such as the magnitude of the velocity disturbance, the required pressure differential resulting from the force set point, the type of actuator (symmetrical or asymmetrical), leakages, the size of the servo valve and the supply pressure. In Figure 6 on the other hand, the operating conditions force the servo valve to operate about valve null when the load cylinders are changing directions. As is clear from Eqs. (13)-(20), this results in abrupt changes in the parameters of the system as the valve goes from positive to negative spool stroke and vice versa. When the controlled output is pressure, the effect of any abrupt changes in the flow gain of the system is intuitively expected to be much more severe than in position or velocity servos due to the continuity equation and the near incompressibility of hydraulic oil, in other words only a minute increase in the flow through the valve may lead to a drastic change in the pressure differential across the actuator in a small amount of time. The effect of abrupt changes in the parameters of a system under linear feedback is equivalent to that of an additional external disturbance that must be attenuated by the feedback (Horowitz, 1963), which makes it clear why a feed forward from the velocity disturbance is not capable of reducing the magnitude of this peaking of the control error.

Using the numerical simulation model it is found that the magnitude of the peaking may be amplified or reduced by adjusting the parameters of Eqs. (13)-(20), as well as the parameters of Eqs. (7)-(8). Furthermore, the magnitude and shape of the peaking may be affected in varying degrees by nonlinearities commonly found in hydraulic servo systems such as hysteresis, backlash, variable gain in the flow characteristics of the servo valve and valve deadband. Figure 7 shows the control error under conditions identical to that of Figure 6 with the exception of the introduction of a minute valve deadband of $0.5 \%$, which may occur in practice even for critically lapped servo valves (Merritt, 1967). In Figure 7 the control error has increased to $2816 \mathrm{~N}$ and $2185 \mathrm{~N}$ without and with the use of feed forward, respectively. Again, as in Figure 6, the 
use of feed forward from the velocity disturbance appears effective in reducing the control error, except at the instances where the actuator is forced to change directions. Although valve deadband may be easily be compensated for, compensation of the other aforementioned nonlinearities is more difficult to achieve and typically constitute uncertain parameters that may be problematic to identify or measure in practice.

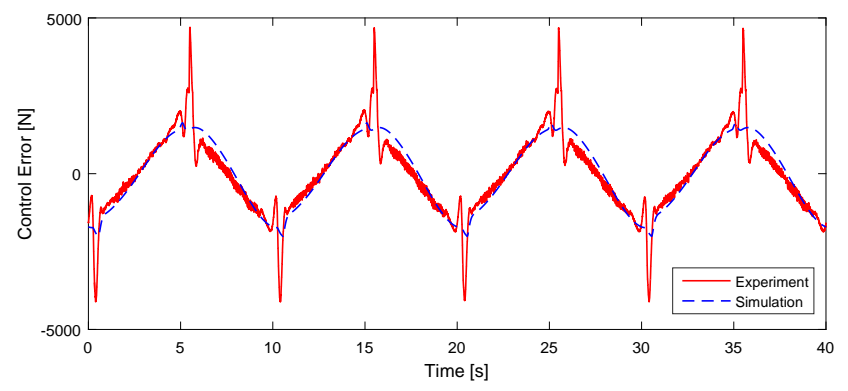

Figure 8: Initial comparison.

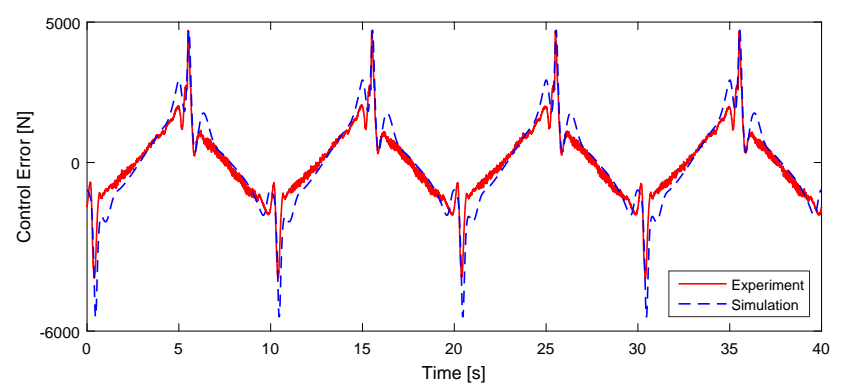

Figure 9: Variable gain characteristics introduced.

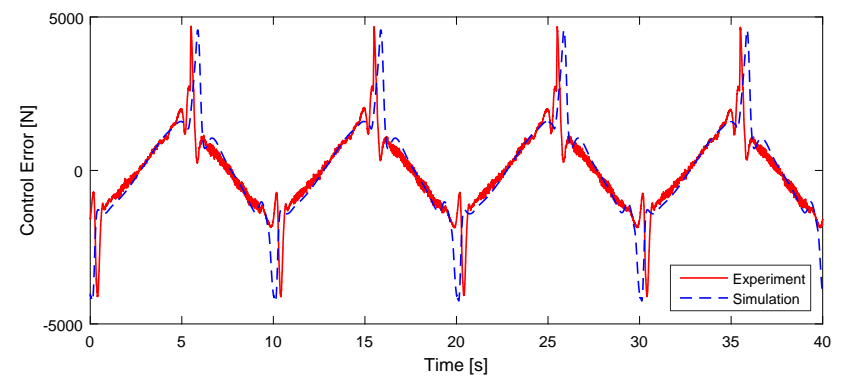

Figure 10: Variable gain characteristics adjusted further.

At this point it is clear that depending upon a number of parameters which may be difficult to predict beforehand, the nonlinear peaking phenomenon may or may not occur in a hydraulic pressure servo subjected to external velocity disturbances and could potentially be amplified by the presence of nonlinearities commonly found in hydraulic systems. Care should be taken to investigate the effects of such nonlinearities when attempting to predict the performance of a system to be designed based on numerical simulations. Figure 8 shows a comparison between the initially expected performance of the experimental system based on numerical simulations and the actual performance in the presence of a sinusoidal velocity disturbance with an amplitude of $40 \mathrm{~mm}$ and a frequency of $0.1 \mathrm{~Hz}$ without the use of velocity feed forward for a force set point of $10 \mathrm{kN}$ using the PI controller of Section 5. Based on the simulations in Figure 8, a minor peak is expected as the load cylinders are forced to change directions. On the experimental system on the other hand, a much larger peak along with two minor peaks are observed that significantly degrade the performance of the system.

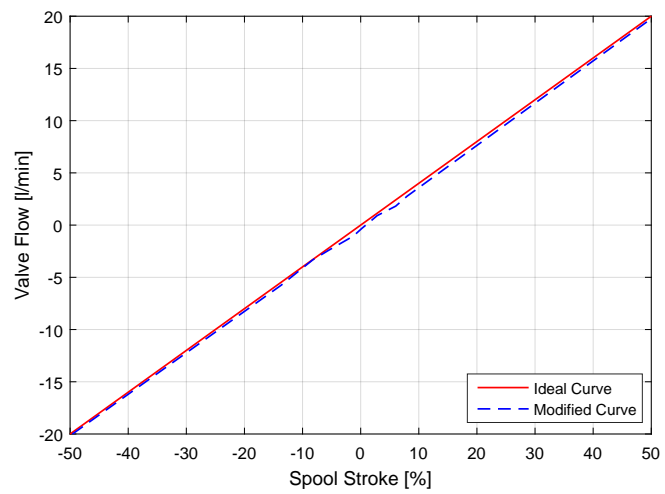

Figure 11: Variable gain characteristics utilized in Figure 9 .

Numerical investigations indicated that out of the aforementioned factors and nonlinearities, the increased magnitude of the peak observed experimentally combined with the overall shape of the control error could only be explained for the system under consideration by imperfections in the curve describing the relation between the spool stroke of the valve and its output flow for a fixed pressure differential across the valve. Modifying this curve in the numerical simulation model as shown in Figure 11, where minor fluctuations in the linearity of the curve have been introduced, a closer match between numerical and experimental data is achieved as seen in Figure 9, where the qualitative shape is matched quite well with a slight mismatch in the amplitudes. Adjusting the curve slightly and modifying each metering edge of the valve individually, the results shown in Figure 10 were obtained, where the 
amplitudes are now closer matched, however with the largest peaks slightly out of phase. Possibly a better match could be achieved by further adjustments, however even minor modifications of the curve in Figure 11 can result in large changes in the control error, which makes obtaining a perfect match rather difficult. The results of Figures 9 and 10 are however sufficient to demonstrate the likelihood that the amplification of the control error on the experimental system is largely due to imperfections in the flow-spool stroke curve of the valve, particularly near valve null. Although there is a slight null shift present in the curve of Figure 11, this is not the cause of the amplification of the control error and similar results may be obtained with a curve that does not have such a null shift. Attempting to compensate for the null shift does not affect the magnitude nor the shape of the control error.

In addition to ensuring valve operation only on one side about valve null, i.e. $u>0$ or $u<0$, which depends upon a number of factors that typically do not constitute a part of the design freedom, numerical investigations based on the model used here revealed that for certain system configurations a sinusoidal control error with the absence of the nonlinear peaking phenomenon may be achieved by sufficient crossport leakage, i.e. from $p_{1}$ to $p_{2}$, even for operating conditions where the valve is operating about null. From a design perspective, including an adjustable crossport orifice between the lines of the actuator could therefore be advantageous in the design of pressure controlled hydraulic actuators subject to external velocity disturbances. Such an orifice could possibly also be used for some system configurations to shift the operating point of the valve away from null.

In the following sections, the capability of ILC to reduce or eliminate the peaking of the control error that occurs as a result of the nonlinear peaking phenomenon is evaluated.

\section{Control Design I: Initial Evaluation}

As mentioned previously, the control specifications are for the load cylinders to apply a constant force in the presence of velocity disturbances caused by the main cylinder. In particular, the load cylinders should be capable of maintaining a force of $10 \mathrm{kN}$ when the main cylinder is actuated with a sinusoidal velocity profile of frequency of $0.1 \mathrm{~Hz}$ with an amplitude of $40 \mathrm{~mm}$. The minimum acceptable accuracy for this application is an accuracy of $\pm 500 \mathrm{~N}$, where greater accuracies are preferred. Initially a PI controller optimized for disturbance rejection using MATLAB's pidtune was eval- uated on the experimental system. Due to the resonant mode resulting from the presence of a flexible environment it was found favourable to include a first order filter in the design, which increased the achievable gain and bandwidth of the controller.

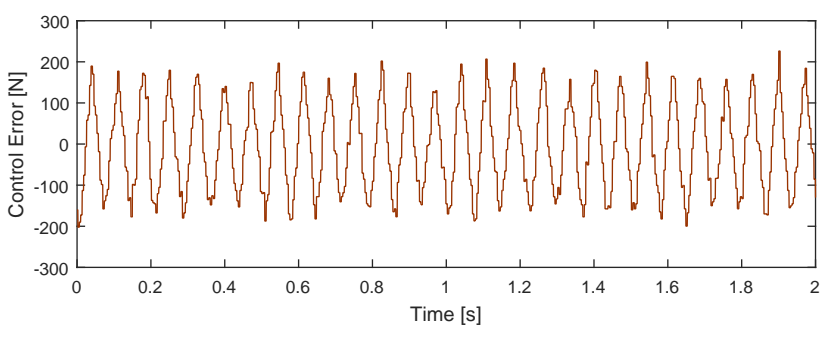

Figure 12: Limit cycle oscillations in the absence of external velocity disturbances.

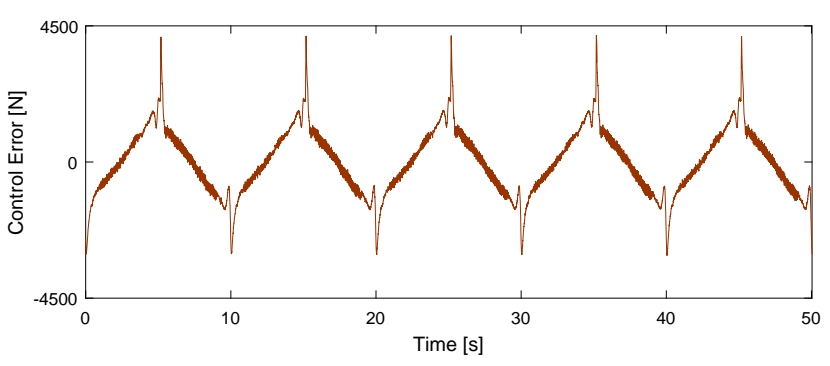

Figure 13: Performance in the presence of velocity disturbance using feedback.

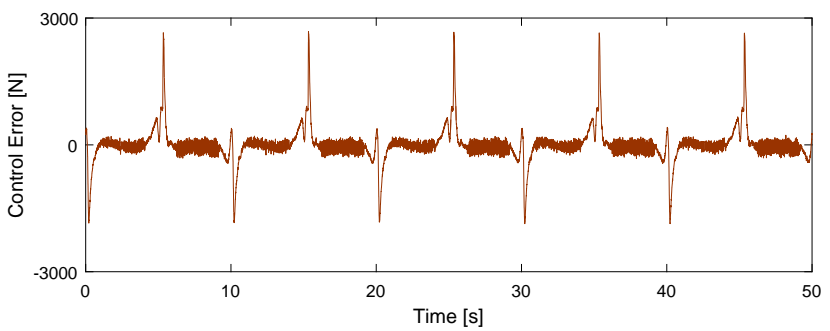

Figure 14: Performance in the presence of velocity disturbance using feedback and feed forward.

Figure 13 shows the performance of the PI controller in the presence of the velocity disturbances generated by the sinusoidal motion of the main cylinder. As pointed out previously, the effects of even minor velocity disturbances can be quite severe due to the near incompressibility of hydraulic oil and the natural velocity feedback as is observed in Figure 13. The presence of the nonlinear peaking phenomenon discussed 
in Section 4 is also evident, where a severe peaking of the control error is observed as the load cylinders are forced to change directions, resulting in a peak-to-peak amplitude of the control error of $7238 \mathrm{~N}$.

The velocity disturbance caused by the main cylinder is measurable, for this reason a fixed gain feed forward from the velocity disturbance was evaluated in combination with the PI controller. Figure 14 shows the resulting control error where it is seen that although the feed forward significantly improves the overall accuracy, the peaking that occurs as a result of the nonlinear peaking phenomenon appears unaffected, resulting in a peak-to-peak amplitude of the error of $4501 \mathrm{~N}$. An explanation for the large magnitude of this peaking was presented in Section 4.

Additionally it was found that the load cylinder circuit inherently suffers from limit cycle oscillations under feedback control, as seen in Figure 12 where a limit cycle with a peak-to-peak amplitude of approximately $400 \mathrm{~N}$ is observed for a constant set point in the absence of external velocity disturbances. The system was also found to limit cycle with a similar amplitude using PID controllers, lag compensators, lead compensators and lag-lead networks. A simple $\mathrm{P}$ controller did not result in limit cycle oscillations, however failed to provide any control over the system with the controlled output simply drifting to an arbitrary value in the absence of velocity disturbances. In the presence of velocity disturbances, the error under $\mathrm{P}$ control reaches its maximum attainable value dictated by pressure relief valves placed in the load cylinder circuit that for safety reasons limit the maximum line pressures. These valves are to be closed under normal operating conditions and were therefore not included in the mathematical model of the system.

\section{Control Design II: Iterative Learning Control}

In general, ILC may be applied alone in an open-loop fashion, or in a closed-loop fashion where the ILC algorithm adjusts the set point or reference trajectory of a feedback controller (Longman, 2000). For the system under consideration, in the absence of a feedback controller, the velocity disturbance generated by the main cylinder is sufficient for the control error to grow until the pressures of the load cylinder circuit reach levels that cause the safety relief valves to activate. The control error then reaches values above $50 \mathrm{kN}$ and the relief valves of the load cylinder circuit are continuously activated. Based on this it was concluded that the application of ILC to the system in an open-loop fashion is not feasible and that ILC must be applied in combination with a feedback controller capable of maintaining a reasonable initial accuracy and preventing the activation of the safety relief valves of the load cylinder circuit. As demonstrated in Section 5 however, the experimental system inherently suffers from limit cycle oscillations under feedback control. This could potentially lead to instability issues due to interactions between the ILC learning algorithm and the limit cycles. Furthermore, even if a stable solution could be achieved in the presence of limit cycles, with the presence of limit cycle oscillations having a peak-to-peak amplitude of $400 \mathrm{~N}$, achievement of a satisfactory accuracy is not a reasonable expectation. For these reasons, a feedback controller is first designed specifically to eliminate limit cycle oscillations, and then applied in combination with ILC.

\subsection{Describing Function Based Feedback Controller}

Numerical investigations traced the cause of the limit cycle oscillations back to the electromagnetic hysteresis of the servo valve. In this section a feedback controller is designed to eliminate limit cycle oscillations due to valve hysteresis based on the describing function of hysteresis. In (Mougenet and Hayward, 1995), the authors proposed based on the general shape of the describing function of hysteresis that its effect may be viewed as a pure phase delay at the crossover frequency and used this to design a feedback controller that successfully eliminated limit cycle oscillations due to valve hysteresis. A similar approach is taken here, where emphasis is also placed on the magnitude increase resulting from the hysteresis nonlinearity. The design approach is therefore to maximize the distance to the critical point for all frequencies near and after crossover in order to prevent intersections with the critical point due to the hysteresis nonlinearity. For a review on limit cycle prediction using describing functions and the describing function of hysteresis, see (Merritt, 1967) and (Franklin et al., 2015).

The controller is designed using the Nichols plot, where the distance to the critical point at any given frequency is easily observed. Figure 15 shows the resulting feedback controller design, where a higher order integrating controller has been designed using a PI controller as a starting point. By cascading multiple lead terms a large radial distance from the critical point is achieved at all frequencies after crossover. The introduction of lead terms brings the resonant mode closer to the critical point, to counteract this effect a complex pole and a complex zero were also placed in the controller to keep the resonant mode at an appropriate distance from the critical point. In this case, the design 
process led to a ninth order controller. Possibly the controller order could be reduced using reduction algorithms, however this was not found to be necessary for implementation on the CompactRIO. This controller is referred to as the describing function based (DFB) feedback controller and its capability to eliminate limit cycle oscillations is evaluated experimentally in Section 7.

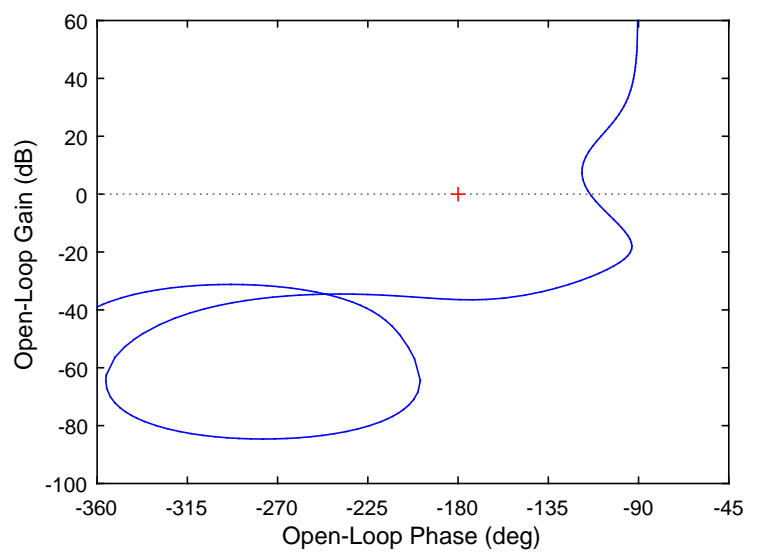

Figure 15: Nichols plot of the DFB feedback controller.

\subsection{Iterative Learning Feed Forward}

The ILC update law considered here is given by (Norrlöf, 2000):

$$
u_{k+1}(t)=u_{k}(t)+L(q) \cdot e_{k}(t)
$$

where $q$ is the discrete time-shift operator and $L(q)$ is a learning filter. The learning filter used in this paper is given by (Wallén, 2011):

$$
L(q)=k \cdot q^{\delta}
$$

where $k$ and $\delta$ are tunable parameters. The parameter $k$ is referred to as the learning gain and is typically chosen to be less than unity (Longman, 2000). For $\delta=0$ the physical interpretation of equation (34) is as follows: at any given discrete point in time, the ILC feed forward is updated by a term that is proportional to the control error observed at the corresponding time step in the previous iteration. For $\delta>0$ the interpretation is similar, except that the ILC feed forward is updated by a term proportional to the control error at $\delta$ time steps ahead of the corresponding time step in the previous iteration. In this manner, the control algorithm may be interpreted as a PD-controller with respect to iteration.

A sufficient condition for monotonic convergence of the ILC is given by (Wallén, 2011):

$$
\left|1-G\left(e^{i \omega \tau}\right) L\left(e^{i \omega \tau}\right)\right|<1
$$

for all $\omega \tau \in[-\pi, \pi]$, where $\tau$ refers to the sample rate and $G$ describes the dynamics of the plant. Equation (35) is referred to here as the stability criterion and may be evaluated graphically on the Nyquist plot by imposing that the Nyquist contour of $G\left(e^{i \omega \tau}\right) L\left(e^{i \omega \tau}\right)$ must be contained inside a unit circle centered at unity in the complex plane (Longman, 2000).

In order to provide robustness towards unmodelled effects such as sensor noise and model uncertainty in the high frequency range, the control error is filtered by a second-order butterworth lowpass filter before sent to the learning filter. This stops the learning process for frequencies above the cutoff frequency of the filter and as a result the convergence criterion has to be fulfilled only for frequencies up to the cutoff frequency (Longman, 2000). For the system at hand it was found by trial and error that a cutoff frequency of $10 \mathrm{~Hz}$ was sufficient to provide long term stability without reducing the convergence rate of the ILC noticeably. The learning gain $\mathrm{k}$ was selected as $k=0.8$, keeping in mind that $k$ is typically selected as less than unity and that larger values of $k$ represent a more aggressive controller (Longman, 2000). The tunable parameter $\delta$ was then adjusted to fulfill the convergence criterion. It was found that $\delta=5$ is sufficient to satisfy the convergence criterion up to and beyond the cutoff frequency and thus provide a stable ILC configuration. For details on selecting the parameters of the ILC learning filter see (Wallén, 2011) and (Norrlöf, 2000). The feedback controller and ILC update law are both implemented using a sample rate of $10 \mathrm{~ms}$.

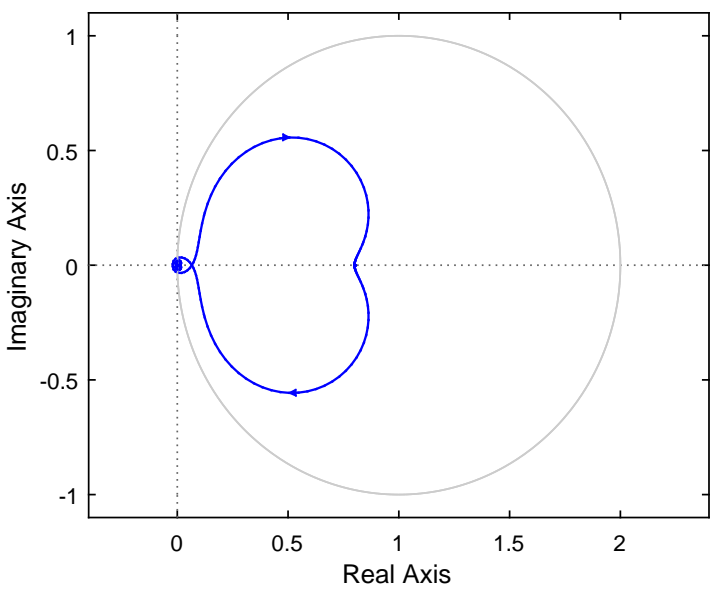

Figure 16: Graphical evaluation of the stability criterion for ILC applied to the DFB feedback controller.

Figure 16 shows the graphical evaluation of the convergence criterion using the Nyquist plot, where the grey circle indicates a unit circle centered at unity. The 
Nyquist contour is contained within the unit circle for frequencies up to about $14 \mathrm{~Hz}$, meaning that the selected cutoff frequency of $10 \mathrm{~Hz}$ is sufficient to achieve a stable ILC configuration. From equation (33) it is seen that the ILC requires storing of the arrays $e_{k}$ and $u_{k}$ in between sampling periods. For the selected sample rate and the system specifications being periodic with a period of 10 seconds this requires the storing of two arrays each containing 1000 elements in between sampling, which is well within the memory capabilities of the CompactRIO. The ILC algorithm is then implemented on the CPU of the CompactRIO together with the DFB feedback controller.

\section{Experimental Results}

Figure 17 shows the control error for a constant set point using the DFB feedback controller in the absence of velocity disturbances, compare with Figure 12 .

It is seen that the DFB feedback controller effectively eliminates limit cycle oscillations in a steady-state situation and the only fluctuations of the control error are due to sensor noise. In Figure 18 the step response of the DFB feedback controller is shown where a reference step change from $9 \mathrm{kN}$ to $10 \mathrm{kN}$ occurs at the 10 second mark. With the exception of minor remnants that may be observed in the transient response for the first two seconds after the step change, limit cycle oscillations have been eliminated in the transient response as well. These minor remnants indicate that the distance to the critical point should not be decreased any further and thus prevents more aggressive tuning of the feedback controller. In addition to the feedback controller, a fixed-gain feed forward from the velocity disturbance is also implemented. Figure 19 shows the control error of this configuration in the presence of the velocity disturbance generated by the main cylinder without the application of ILC. The peak-to-peak amplitude and RMS of the control error are $9197 \mathrm{~N}$ and $1842 \mathrm{~N}$, respectively. The PI controller discussed in Section 5 achieved a peak-to-peak control error of $4501 \mathrm{~N}$ when combined with fixed-gain feedforward, which indicates that the performance of the DFB feedback controller is somewhat conservative. This is however a natural result of the design approach that was taken in order to eliminate limit cycle oscillations, as increasing the distance to the critical point reduces the achievable performance as described by Bode's integral of feedback (Lurie and Enright, 2012).

Next, the ILC algorithm is activated. The initial convergence after activation of the ILC is shown in Figure 20 over two minutes, where the ILC is activated shortly before the 20 second mark. In Figure 20 both the RMS and the peak-to-peak amplitude of the control error are seen to decrease monotonically each iteration. After six minutes and forty seconds, the control error appears to have converged to its final accuracy.

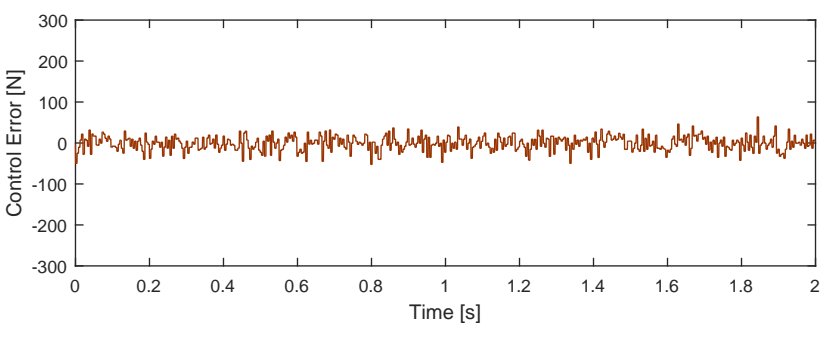

Figure 17: Elimination of limit cycle oscillations, steady-state.

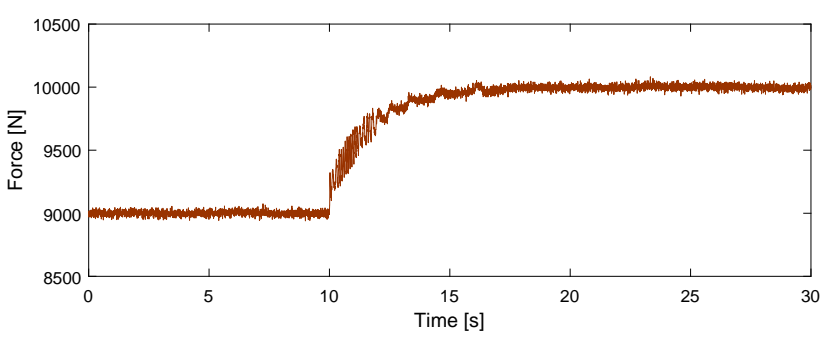

Figure 18: Elimination of limit cycle oscillations, transient response.

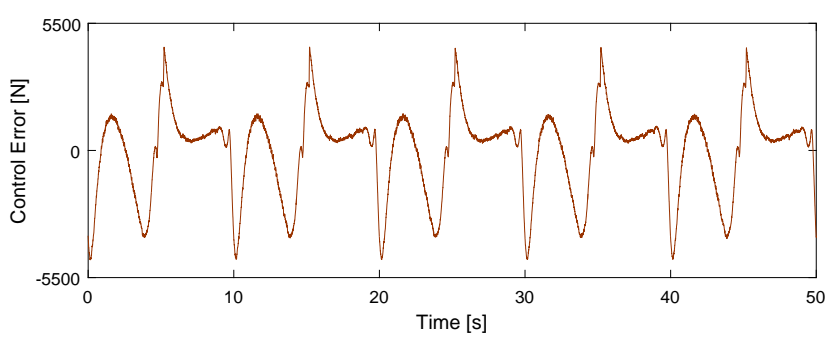

Figure 19: Performance in the presence of velocity disturbance.

Figure 21 shows the control error over eight iterations after convergence where $k_{0}$ indicates the iteration where zero minutes have passed since convergence. The accuracy is maintained within a $\pm 250 \mathrm{~N}$ band for the majority of the time, with a reduction in the peak-topeak amplitude of the control error by a factor of more than 19 and a reduction in the RMS of the error by a factor of more than 30 .

The DFB-ILC combination achieves long term stability and maintains the converged accuracy as time 
grows large. Figure 22 shows the control error two hours after convergence of the ILC where the the converged accuracy is still maintained. This corresponds to the ILC having been active for more than 750 iterations, demonstrating the long term stability of the DFB-ILC combination. Inspecting the control error at other points in time, errors similar to that of Figures 21 and 22 are observed.

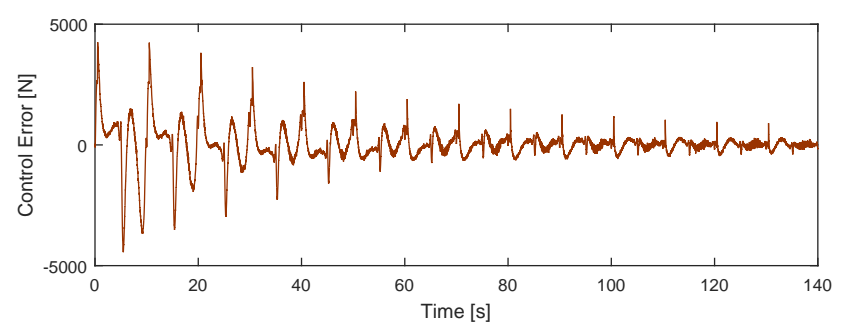

Figure 20: Initial convergence of the ILC.

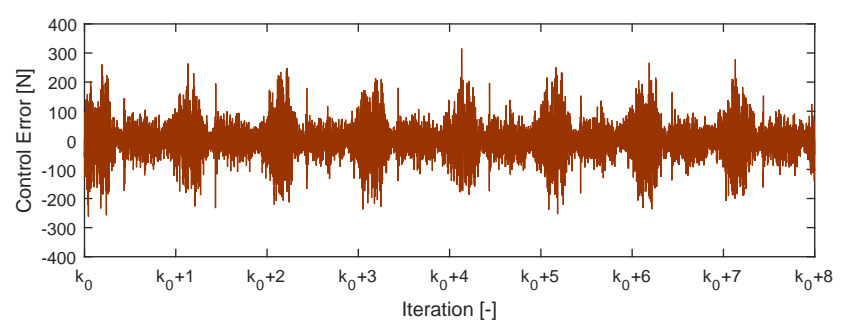

Figure 21: Control error after convergence of the ILC.

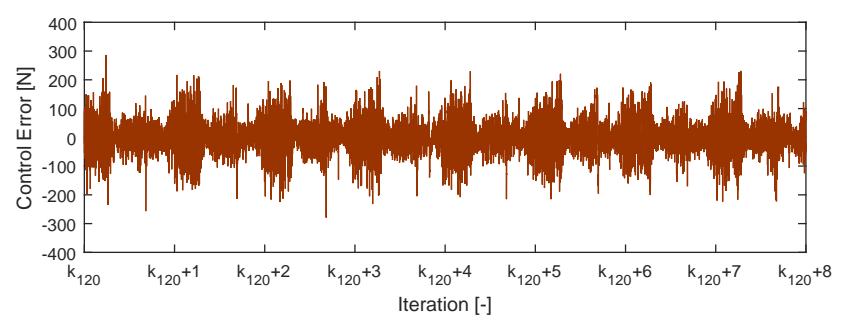

Figure 22: Control error two hours after convergence of the ILC.

It should be noted that the peaking of the control error resulting from the nonlinear peaking phenomenon that is observed in Figure 20 appears to completely disappear as the ILC converges. The maximum amplitude of the converged control error seen in Figures 21 occurs when the cylinders are moving with their maximum velocity, whereas the nonlinear peaking phenomenon occurs when the cylinders are close to zero velocity. In other words, the nonlinear peaking phenomenon is no longer the limiting factor in terms of performance, and it may be concluded that its effects have been eliminated by the application of ILC.

\section{Discussion and Conclusions}

In this paper a performance limiting phenomenon that may occur in the pressure control of hydraulic actuators was presented and investigated. It was shown that depending upon the system configuration and operating conditions, a severe peaking of the control error may be observed in the presence of external velocity disturbances. Numerical investigations showed that the occurrence of such a phenomenon depends upon a number of factors that may be difficult to predict for a given system without access to experimental data. The significance of the phenomenon from a design perspective and possible ways of avoiding it were discussed. For situations where the peaking of the control error cannot be avoided, ILC was proposed and evaluated as a solution for improving the system's performance. For the system under consideration, application of ILC in an open-loop fashion was not feasible due to the presence of external velocity disturbances, necessitating the application of ILC in combination with a feedback controller. The experimental system was found to inherently suffer from limit cycle oscillations under feedback control due to the presence of valve hysteresis. For this reason a feedback controller was designed for the elimination of limit cycle oscillations based on the describing function of hysteresis and applied in combination with the ILC.

Experimental results demonstrate the elimination of limit cycle oscillations and long term stability of the proposed solution, evaluated here for more than 750 iterations of continuous operation throughout which the ILC learning algorithm remained active. Upon convergence of the ILC the maximum amplitude of the control error remains less than $0.3 \mathrm{kN}$, with the RMS and peak-to-peak of the error having been reduced by factors of 30 and 19, respectively. Complete convergence was achieved in 40 iterations, however an accuracy greater than $0.5 \mathrm{kN}$ was achieved already after 23 iterations.

Several accounts of ILC applied to hydraulic motion control problems have appeared in the literature, see for example (Lingjun et al., 2014), (Chen and Zeng, 2003), (Daley et al., 2004) and (Zhao et al., 2005), where the achieved reduction in the control error vary significantly from one application to another, even for similar or identical ILC algorithms. The reported convergence rates in these applications range from anywhere between 15 and 100 iterations, also for similar 
algorithms. Presumably the achievable improvement using a given ILC algorithm depends upon a number of factors such as the system configuration, tuning of the ILC algorithm, whether applied in an open-loop fashion or a closed-loop fashion as well as the feedback controller when applied in a closed-loop fashion.

Previous investigations of ILC applied to hydraulic pressure control problems on the other hand appear to be limited, where a previous application suitable for comparison has been located in (Wang et al., 2015), where ILC was applied to improve the tracking accuracy of a pressure controlled hydraulic actuator in the absence of external velocity disturbances. Limit cycle oscillations were not present and the absence of velocity disturbances allowed the application of ILC both in an open-loop and closed-loop fashion, the latter resulting in the better performance. Improved tracking accuracy was achieved for both low and high frequency tracking, where the high frequency tracking achieved an accuracy of $0.5 \mathrm{kN}$, improving the accuracy by a factor of four over a conventional PID controller after 25 iterations. This is comparable to the results presented in this paper, with the exception of the factor by which ILC improved the accuracy. This difference is likely due to a larger initial error resulting from the presence of the nonlinear peaking phenomenon as well as limit cycle oscillations which necessitated the use of a rather conservative feedback controller.

In summary the results presented here compare well to that of previous applications both in terms of accuracy and convergence rate, the novelty of the results presented here being the application of ILC to eliminate the effects of the nonlinear peaking phenomenon and the application of ILC in a closed-loop fashion to a system that inherently suffers from limit cycle oscillations under feedback. By applying ILC in combination with the DFB feedback controller both the effects of the nonlinear peaking phenomenon and limit cycle oscillations were eliminated and stability was achieved with a satisfactory accuracy for long periods of continuous operation. For the system under consideration the convergence rate is also satisfactory. Faster convergence may possibly be achieved using a stronger feedback controller, however preliminary attempts to accomplish this were not successful due to the appearance of limit cycles when increasing the gain of the controller or decreasing the distance to the critical point. Further investigations into feedback controller design for the elimination of limit cycle oscillations in valve controlled hydraulic actuators will be the topic of future research.

\section{Acknowledgments}

The research presented in this paper has received funding from the Norwegian Research Council, SFI Offshore Mechatronics, project number 237896.

\section{References}

Baghestan, K., Rezaei, S., Talebi, H., and Zareinejad, M. Robust force control in a novel electrohydraulic structure using polytopic uncertainty representation. ISA transactions, 2014. 53(6):18731880. doi:10.1016/j.isatra.2014.08.002.

Blanken, L., Boeren, F., Bruijnen, D., and Oomen, T. Batch-to-batch rational feedforward control: from iterative learning to identification approaches, with application to a wafer stage. IEEE/ASME Transactions on Mechatronics, 2017. 22(2):826-837. doi:10.1109/TMECH.2016.2625309.

Boeren, F., Bareja, A., Kok, T., and Oomen, T. Frequency-domain ILC approach for repeating and varying tasks: With application to semiconductor bonding equipment. IEEE/ASME Transactions on Mechatronics, 2016. 21(6):2716-2727. doi:10.1109/TMECH.2016.2577139.

Chen, C.-K. and Zeng, W.-C. The iterative learning control for the position tracking of the hydraulic cylinder. JSME International Journal Series $C$ Mechanical Systems, Machine Elements and Manufacturing, 2003. 46(2):720-726. doi:10.5739/isfp.2002.591.

Conrad, F. and Jensen, C. Design of hydraulic force control systems with state estimate feedback. In Proceedings of the IFAC 10th Triennial Congress. pages 307-312, 1987. doi:10.1016/S1474-6670(17)55388-4.

Daley, S., Hatonen, J., and Owens, D. Hydraulic servo system command shaping using iterative learning control. In Proc. of the Control Conf. pages 117121, 2004. doi:10.1049/ic:20040657.

Esfandiari, M. and Sepehri, N. On high bandwidth output pressure control design of hydraulic actuators using quantitative feedback theory. Transactions of the Canadian Society for Mechanical Engineering, 2014. 38(4):533-555. doi:10.1631/FITEE.1601104.

Franklin, G. F., Powell, J. D., and Abbas, E.-N. Feedback control of dynamic systems. Pearson, 2015.

Gøytil, P. H. Closed-Loop Electrohydraulic Pressure Control. University of Agder, MSc thesis, 2017. 
Horowitz, I. Synthesis of Feedback Systems. Academic Press, 1963.

Jiao, Z.-X., Gao, J.-X., Qing, H., and Wang, S.-P. The velocity synchronizing control on the electrohydraulic load simulator. Chinese journal of aeronautics, 2004. 17(1):39-46. doi:10.1016/S10009361(11)60201-X.

Klausen, A. and Tørdal, S. S. System Identification of a Variable Piston Pump and Design of a Hydraulic Load Circuit. University of Agder, MSc thesis, 2015.

Lamming, C., Plummer, A. R., and Hillis, A. J. Analysis of robust electrohydraulic force control. In 7th International Fluid Power Conference. Achen, Germany, 2010.

Ledezma, J. A., De Negri, V. J., and De Pieri, E. R. New approach for hydraulic force control based on hydraulic compliance. In International Conference on Fluid Power and Mechatronics. pages 454-459, 2015. doi:10.1109/FPM.2015.7337160.

Lingjun, L., Poms, U., and Thurner, T. Accurate position control of a servo-hydraulic test cylinder by iterative learning control technique. In European Modelling Symposium. pages 297-302, 2014. doi:10.1109/EMS.2014.87.

Longman, R. W. Iterative learning control and repetitive control for engineering practice. International journal of control, 2000. 73(10):930-954. doi:10.1080/002071700405905.

Lurie, B. J. and Enright, P. J. Classical feedback control: with matlab and simulink. CRC Press, 2012.

Merritt, H. E. Hydraulic control systems. John Wiley \& Sons Inc., 1967.
Mougenet, F. and Hayward, V. Limit cycle characterization, existence and quenching in the control of a high performance hydraulic actuator. In IEEE Int. Conf. Robot. Automat., volume 3. pages 2218-2223, 1995. doi:10.1109/ROBOT.1995.525591.

Norrlöf, M. Iterative Learning Control: Analysis, Design, and Experiments. Linköping University, $\mathrm{PhD}$ thesis, 2000.

Ottestad, M., Nilsen, N., and Hansen, M. R. Reducing the static friction in hydraulic cylinders by maintaining relative velocity between piston and cylinder. In 12th International Conference on Control, Automation and Systems. pages 764-769, 2012.

Wallén, J. Estimation-based iterative learning control. Linköping University, PhD thesis, 2011.

Wang, S.-k., Zhao, J.-b., and Wang, J.-z. Openclosed-loop iterative learning control for hydraulically driven fatigue test machine of insulators. Journal of Vibration and Control, 2015. 21(12):22912305. doi:10.1177/1077546313508998.

Zhao, J., French, C., Shield, C., and Posberg, T. Effective force testing with nonlinear velocity feedback compensation. In 13th World Conference on Earthquake Engineering. Vancouver, Canada, 2004.

Zhao, S., Wang, J., Wang, L., Hua, C., and He, Y. Iterative learning control of electro-hydraulic proportional feeding system in slotting machine for metal bar cropping. International Journal of $M a-$ chine Tools and Manufacture, 2005. 45(7):923-931. doi:10.1016/j.ijmachtools.2004.10.013. 